\title{
Increase of Bovine Fascioliasis in Puerto Rico as Determined by Slaughterhouse Surveys ${ }^{1}$
}

\author{
Anne D. Frame, Pedro Bendezú, Héctor Mercado, Hugo Otiniano, \\ Stephen J. Frame, and Waldemar Flores ${ }^{2}$
}

\begin{abstract}
Surveys conducted at all the existing slaughterhouses in Puerto Rico during the period of 1973-76 showed that the prevalence of Fasciola hepatica in cattle steadily increased from $23.83 \%$ in 1973 to $31.76 \%$ in 1976 . This increase was found to be even more dramatic when compared to the 1948-49 survey, where only $7.55 \%$ of the bovine livers were found to be infected. For the period of $1973-76,1.76$ million pounds of bovine livers were condemned. This represents a direct loss of over 1.584 million dollars for the Puerto Rican cattlemen in condemned livers alone, and multiple indirect losses for the local economy.
\end{abstract}

\section{INTRODUCTION}

The first large scale survey of fascioliasis in cattle in Puerto Rico was documented by Rivera Anaya and Martínez de Jesús in 1948-49 (5). They surveyed the 38 slaughterhouses in Puerto Rico and found that the prevalence of this bovine disease was $7.55 \%$. In 1973 Chiriboga et al. (1), in a limited survey of 9 slaughterhouses, observed an increase in this infection. Since that time we are unaware of any published report on the status of Fasciola hepatica throughout the Island. In this study we report a survey of all of the slaughterhouses in Puerto Rico with respect to prevalence of $F$. hepatica in cattle. In addition, we discuss some of the economic impact of this infection in the Island. A brief report on these results has been published by Frame and Bendezú (4).

\section{MATERIALS AND METHODS}

With the cooperation of the Department of Agriculture, data were obtained regarding the number of cattle slaughtered per month at all the existing licensed abattoirs in Puerto Rico during 1973-76. Of the animals slaughtered, the number infected with $F$. hepatica, as determined by condemned livers, was obtained. In addition, 76 condemned livers from infected cows were weighed for an approximation of the amount of edible food lost due to the infection.

\section{RESULTS AND DISCUSSIONS}

Table 1 summarizes the number of cattle slaughtered at each of the operating abattoirs in Puerto Rico during 1973-76, together with the

${ }^{1}$ Manuscript submitted to Editorial Board March 31, 1978.

${ }^{2}$ Department of Natural Sciences, Hato Rey Campus, Inter-American University, San Juan, P.R. 
TABLE 1.-Prevalence of Fasciola hepatica in Puerto Rico as determined by livers condemned at the slaughterhouses. Data obtained in 1973-76 is compared with that of 1948-49

\begin{tabular}{|c|c|c|c|c|c|c|c|c|c|c|c|c|c|c|c|}
\hline \multirow{3}{*}{$\begin{array}{l}\text { Slaughter- } \\
\text { house }\end{array}$} & \multicolumn{3}{|c|}{$1948-49$} & \multicolumn{3}{|c|}{1973} & \multicolumn{3}{|c|}{1974} & \multicolumn{3}{|c|}{1975} & \multicolumn{3}{|c|}{1976} \\
\hline & \multicolumn{2}{|c|}{ Animals } & \multirow{2}{*}{$\begin{array}{l}\% \text { in- } \\
\text { fected }\end{array}$} & \multicolumn{2}{|c|}{ Animals } & \multirow{2}{*}{$\begin{array}{l}\% \text { in- } \\
\text { fected }\end{array}$} & \multicolumn{2}{|c|}{ Animals } & \multirow{2}{*}{$\begin{array}{l}\% \text { in- } \\
\text { fected }\end{array}$} & \multicolumn{2}{|c|}{ Animals } & \multirow{2}{*}{$\begin{array}{l}\% \text { in- } \\
\text { fected }\end{array}$} & \multicolumn{2}{|c|}{ Animals } & \multirow{2}{*}{$\begin{array}{l}\% \text { in- } \\
\text { fected }\end{array}$} \\
\hline & $\begin{array}{l}\text { Sacri- } \\
\text { ficed }\end{array}$ & Infected & & $\begin{array}{l}\text { Sacri- } \\
\text { ficed }\end{array}$ & Infected & & $\begin{array}{l}\text { Sacri- } \\
\text { ficed }\end{array}$ & Infected & & $\begin{array}{l}\text { Sacri- } \\
\text { ficed }\end{array}$ & Infected & & $\begin{array}{l}\text { Sacri- } \\
\text { fied }\end{array}$ & Infected & \\
\hline Aguadilla & 1,638 & 226 & 13.8 & 2,174 & 428 & 19.69 & 3,534 & 764 & 21.65 & 3,643 & 737 & 20.23 & 1,733 & 543 & 31.33 \\
\hline Aibonito & 596 & 27 & 4.53 & 1,461 & 388 & 26.56 & 1,587 & 334 & 21.05 & 616 & 176 & 28.57 & 957 & 348 & 36.36 \\
\hline Arecibo & 2,583 & 273 & 10.50 & 12,108 & 2,248 & 18.16 & 9,542 & 2,198 & 23.00 & 8,846 & 2,482 & 28.05 & 7,066 & 2,592 & 26.86 \\
\hline Cabo Rojo & - & - & - & - & - & - & 3,332 & 559 & 16.78 & 4,339 & 735 & 16.94 & 3,779 & 801 & 21.20 \\
\hline Caguas & 4,248 & 73 & 1.70 & 26,085 & 6,172 & 23.70 & 21,016 & 6,515 & 31.00 & - & - & - & - & - & - \\
\hline Carolina & - & - & - & 9,218 & 3,015 & 32.71 & 7,530 & 2,274 & 30.20 & 5,419 & 2,290 & 42.25 & 7,412 & 3,508 & 47.46 \\
\hline Corozal & 585 & 20 & 3.40 & 9,176 & 2,561 & 27.90 & 6,458 & 1,982 & 30.70 & 6,095 & 2,398 & 39.34 & 2,886 & 1,483 & 51.38 \\
\hline Humacao & 2,504 & 143 & 5.71 & 4,483 & 998 & 22.26 & 6,324 & 1,316 & 20.81 & 5,524 & 998 & 18.07 & 6,184 & 1,317 & 21.30 \\
\hline Juana Diaz & - & - & - & 4,520 & 646 & 14.29 & 1,429 & 182 & 12.74 & 2,072 & 397 & 19.16 & 4,481 & 867 & 19.35 \\
\hline Lares & 578 & 70 & 12.11 & - & - & - & - & - & - & - & - & - & 1,293 & 542 & 41.92 \\
\hline Manati & 251 & 145 & 57.70 & 5,568 & 1,571 & 28.21 & 6,115 & 1,353 & 22.10 & 3,420 & 1,409 & 41.20 & 3,578 & 1,571 & 43.80 \\
\hline Mayagüez & 6,914 & 745 & 10.77 & - & - & - & 3,821 & 684 & 17.90 & 6,331 & 1,003 & 15.84 & 7,721 & 1,402 & 18.16 \\
\hline Naguabo & 688 & 37 & 5.30 & 8,039 & 2,718 & 33.81 & 6,363 & 2,159 & 33.91 & 5,511 & 1,931 & 35.03 & 5,155 & 2,246 & 43.51 \\
\hline Ponce & 7,338 & 133 & 1.81 & 2,748 & 593 & 21.57 & 4,728 & 799 & 16.89 & 4,092 & 718 & 17.54 & 2,907 & 543 & 18.68 \\
\hline Quebradillas & - & - & - & 1,375 & 235 & 17.09 & 1,482 & 279 & 18.83 & 1,670 & 422 & 25.27 & 953 & 208 & 21.83 \\
\hline San Germán & 1,349 & 161 & 11.93 & - & - & - & - & - & - & 1,740 & 426 & 24.48 & 2,152 & 541 & 25.14 \\
\hline San Juan & 2,638 & 356 & 13.50 & 13,517 & 3,446 & 25.50 & 5,323 & 1,379 & 25.91 & 8,966 & 1,947 & 32.87 & 13,853 & 4,493 & 32.24 \\
\hline $\begin{array}{l}\text { San Seba- } \\
\text { stián }\end{array}$ & - & - & - & 1,489 & 519 & 34.85 & 1,319 & 514 & 38.96 & 1,206 & 439 & 36.40 & 1,087 & 454 & 41.77 \\
\hline Utuado & 1,387 & 204 & 14.70 & 4,850 & 233 & 4.80 & 7,441 & 284 & 3.80 & 1,191 & 380 & 31.90 & 775 & 283 & 36.52 \\
\hline $\begin{array}{l}\text { Yauco } \\
\text { Others }\end{array}$ & - & - & - & 2,745 & 408 & 14.86 & 1,505 & 232 & 15.41 & 907 & 203 & 22.38 & 1,779 & 317 & 17.82 \\
\hline TOTALS & $46,565^{3}$ & 3,515 & 7.55 & 109,556 & 26,179 & 23.90 & 98,849 & 23,808 & 24.09 & 71,588 & 20,091 & 28.06 & 75,751 & 24,059 & 31.76 \\
\hline
\end{tabular}

'Slaughterhouse closed.

${ }^{2}$ Other slaughterhouses surveyed in 1948-49 not opened during our study not included.

${ }^{3}$ Total taken from Rivera Anaya and Martinez de Jesús 1948-49 report (5). 
number of animals infected. In addition, we report the results published by Rivera Anaya and Martinez de Jesús for the period 1948-49 (5) for comparison.

The prevalence of $F$. hepatica increased steadily, and of the 20 slaughterhouses surveyed, 16 showed an increase in infected cattle over previous years.

Although an accurate estimate of the economic losses to the cattle industry in Puerto Rico due to $F$. hepatica is not easy to make, it has been estimated that at least one million dollars is lost to the Puerto Rican economy annually (3).

Indirect losses due to reduction in milk and meat production are difficult to determine. Ross (6) calculated an $8 \%$ reduction in milk production per cow with a low grade infection; he also estimated that there was a similar reduction in weight. Direct losses due to liver condemnation, however, can be measured. De León, Ritchie, and Chiriboga (2) reported a direct loss of at least 600,000 dollars due to infested livers in the Island from January to June 1969. Table 2 summarizes the direct losses due to condemned livers for the period 1973-76. We weighed 76 infected livers and found them to have a mean weight of $18.7 \mathrm{lb}$. In November 1977 reliable meat dealers reported the wholesale price of fresh local beef liver at around $\$ 0.90$ per pound, and as much as $\$ 1.29$ at the retail level. These figures reveal a direct loss of $\$ 1,584,000.00$ to the cattle industry, due to condemned livers alone, for the period 1973-76 and a loss of over $\$ 2,270,000.00$ from the local economy at the retail level.

This increase in bovine fascioliasis in Puerto Rico, coupled with the decrease of cattle slaughtered, should be reason for concern.

\section{RESUMEN}

Encuestas realizadas en todos los mataderos en Puerto Rico durante el período de 1973-76 demostraron que la prevalencia de Fasciola hepatica en el ganado aumentó firmemente de 23.83 por ciento en 1973 a 31.76 en 1976. Este incremento fue aún más dramático al compararse con los resultados de la encuesta del período 1948-49, en la cuâl se informa que solamente el 7.55\% de hígados vacunos estaban infectados. Para el período de 1973-76 sobre 1.76 millones de libras de hígados vacunos fueron desechadas. Esto representa

TABLE 2.-Estimate of direct losses due to condemned livers infected with Fasciola hepatica for the period 1973-76

\begin{tabular}{crrcr}
\hline Year & $\begin{array}{c}\text { Total cattle sac- } \\
\text { rificed }\end{array}$ & $\begin{array}{c}\text { Total cattle } \\
\text { infected }\end{array}$ & $\begin{array}{c}\text { Total livers condemned } \\
(18.7 \text { mean weight) }\end{array}$ & $\begin{array}{c}\text { Losses due to condemned } \\
\text { livers at wholesale price } \\
\text { of } \$ 0.90\end{array}$ \\
\hline & & & $l b$ & \\
1973 & 109,556 & 26,179 & $489,546.30$ & $\$ 440,591.67$ \\
1974 & 98,849 & 23,808 & $445,209.60$ & $400,688.64$ \\
1975 & 71,588 & 20,091 & $375,701.70$ & $338,131,53$ \\
1976 & 75,751 & 24,059 & $449,903.30$ & $404,912.97$ \\
Total & 355,744 & 94,137 & $1,760,361.90$ & $1,584,325.70$ \\
\hline
\end{tabular}


una pérdida directa de sobre 1.584 millones de dólares para los ganaderos puertorriqueños y múltiples pérdidas indirectas para la economía local.

\section{LITERATURE CITED}

I. Chiriboga, et al., 1973. Unpublished data.

2. De León, D., Ritchie, L. S., and Chiriboga, J., 1972. Fascioliasis in dairy cattle in Río Plata basin of the Dorado Area, P.R., J. Agri. Univ. P.R. 56 (1): 82-92.

3. De León, D., Ritchie, L. S., and Chiriboga, J., PRNC-148, Programa de Fasciola hepatica en Puerto Rico. Primer Informe, enero de 1970 a abril de 1971.

4. Frame, Anne and Bendezú, P., 1978. Bovine fascioliasis in Puerto Rico. J. Parasitol. Vol 64 (1), res. note.

5. Rivera Anaya, J. D. and Martinez de Jesús, J., 1952. The extent of liver-fluke infestation of cattle in Puerto Rico (a slaughterhouse survey). Agri. Exp. Stn. Univ. P.R., Bull. 107.

6. Ross, J. G., 1970. The economics of Fasciola hepatica infections in cattle. Br. Vet. J. 126 XIII. 